\title{
A small molecule targeting protein translation does not rescue spatial learning and memory deficits in the hAPP-J20 mouse model of Alzheimer's disease
}

\author{
Erik C.B. Johnson ${ }^{\text {Corresp., }}{ }^{1,2}$, Jing Kang ${ }^{1}$ \\ ${ }^{1}$ Gladstone Institute of Neurological Disease, Gladstone Institutes, San Francisco, California, United States \\ 2 Department of Neurology, University of California - San Francisco, San Francisco, California, United States \\ Corresponding Author: Erik C.B. Johnson \\ Email address: erik.johnson@gladstone.ucsf.edu
}

A small molecule named ISRIB has recently been described to enhance memory in rodents. In this study we aimed to test whether ISRIB would reverse learning and memory deficits in the J20 mouse model of human amyloid precursor protein (hAPP) overexpression, a model that simulates many aspects of Alzheimer's disease in which memory deficits are a hallmark feature. We did not observe a significant rescue effect with ISRIB treatment on spatial learning and memory as assessed in the Morris water maze in J20 mice. We also did not observe a significant enhancement of spatial learning or memory in nontransgenic mice with ISRIB treatment, although a trend emerged for memory enhancement in one cohort of mice. Future preclinical studies with ISRIB would benefit from additional robust markers of target engagement in the brain. 


\section{A Small Molecule Targeting Protein Translation Does Not Rescue Spatial Learning and Memory Deficits in the hAPP-J20 Mouse Model of Alzheimer's Disease}

Erik C.B. Johnson ${ }^{\ddagger \ddagger}$, Jing Kang ${ }^{\dagger}$

† Gladstone Institute of Neurological Disease, Gladstone Institutes, 1650 Owens Street, San Francisco, California 94158, USA

‡ Department of Neurology, University of California - San Francisco, 675 Nelson Rising Lane, San Francisco, California 94158, USA

Corresponding Author: Erik C.B. Johnson ${ }^{\dagger \ddagger}$

Email address: erik.johnson@gladstone.ucsf.edu 


\section{Abstract}

2 A small molecule named ISRIB has recently been described to enhance memory in rodents. In

3 this study we aimed to test whether ISRIB would reverse learning and memory deficits in the J20

4 mouse model of human amyloid precursor protein (hAPP) overexpression, a model that

5 simulates many aspects of Alzheimer's disease in which memory deficits are a hallmark feature.

6 We did not observe a significant rescue effect with ISRIB treatment on spatial learning and

7 memory as assessed in the Morris water maze in J20 mice. We also did not observe a significant

8 enhancement of spatial learning or memory in nontransgenic mice with ISRIB treatment,

9 although a trend emerged for memory enhancement in one cohort of mice. Future preclinical

10 studies with ISRIB would benefit from additional robust markers of target engagement in the

11 brain.

\section{Introduction}

14 The prevalence of Alzheimer's disease (AD) is increasing as the human population ages, and

15 treatments that slow or reverse the disease are urgently needed (Alzheimer's, 2015). In AD, loss

16 of the ability to form new memories, and eventually to recall long-term memories, is a defining

17 clinical feature. While the causes of AD remain a focus of intense investigation, treatments that

18 can enhance the brain's innate ability to form and retrieve memories, or that counteract the mechanisms the lead to memory loss, could offer an immediate benefit to the millions of people

20 who currently suffer from this disease.

21 Recently, a symmetric bis-glycolamide named ISRIB (integrated stress response inhibitor) has

22 been described to enhance spatial and fear memory in rodents when delivered immediately after 
a stimulus or completion of a behavioral task (Sidrauski et al., 2013). ISRIB binds to and activates the guanine nucleotide exchange factor eIF2B (elongation initiation factor $2 \mathrm{~B}$ ), which in turn relieves inhibition of protein translation caused by phosphorylation of the alpha subunit of initiation factor 2 (eIF2 $\alpha$ ) (Sidrauski et al., 2013; Sekine et al., 2015; Sidrauski et al., 2015a; Sidrauski et al., 2015b). While phosphorylation of eIF2 $\alpha$ is a key event in the integrated stress response (ISR), in which a diverse array of cellular stressors can lead to an overall decrease in the rate of protein translation (Walter and Ron, 2011; Halliday and Mallucci, 2015), baseline phosphorylation levels of eIF $2 \alpha$ also provide a homeostatic "brake" on global protein translation rates even in the absence of cellular stress and activation of the ISR. Release of this brake through pharmacological activation of eIF2B by ISRIB has been proposed to lead to enhanced memory formation, possibly through enhancement of cAMP responsive element-binding protein (CREB) expression (Costa-Mattioli et al., 2007; Stern et al., 2013), a protein essential for memory formation (Kida and Serita, 2014), or through other mechanisms such as modulation of synaptic long-term depression (LTD) (Di Prisco et al., 2014).

Mouse models in which the human amyloid precursor protein (hAPP) is overexpressed in the brain have been widely used as models of AD (Webster et al., 2014). The hAPP-J20 (J20) mouse model contains the Swedish and Indiana hAPP mutations that cause familial AD, leading to overproduction of total levels of amyloid-beta $(A \beta)$ as well as an increase in the A $\beta 1-42 / A \beta 1$ 40 ratio (Mucke et al., 2000). The $\mathrm{J} 20$ model recapitulates many aspects of $\mathrm{AD}$, including the formation of aggregated $A \beta$ in the brain and the development of deficits in learning and memory (Mucke et al., 2000; Webster et al., 2014). Spatial learning and memory, as assessed by performance in the Morris water maze (Morris, 1984), is robustly impaired in J20 (Roberson et al., 2007; Sanchez-Mejia et al., 2008; Cisse et al., 2011; Sanchez et al., 2012). The precise 
46 mechanism(s) underlying this impairment remains under investigation, but one such mechanism

47 may be enhanced LTD. LTD has been shown to be pathologically elevated in the hippocampus

48 in the presence of aggregated forms of $A \beta$ (Shankar et al., 2008; Luscher and Huber, 2010;

49 Pozueta et al., 2013). Treatment with ISRIB prevents the transcriptional program required for

50 induction of LTD (Sidrauski et al., 2013; Di Prisco et al., 2014), and may thereby provide an

51 effective treatment for spatial learning and memory problems in both the $\mathrm{J} 20$ model and in

52 patients with AD.

53 In this preclinical study, we tested whether ISRIB could enhance not only spatial learning and

54 memory and fear memory in nontransgenic mice through a mechanism that remains to be

55 completely defined (Sidrauski et al., 2013; Stern et al., 2013), but also whether it similarly could

56 improve spatial learning and memory deficits in the J20 AD mouse model. In our experimental

57 design, we made an effort to replicate as closely as possible the dosing regimens and behavioral

58 protocols used in the prior study describing enhanced memory performance with ISRIB

59 treatment (Sidrauski et al., 2013).

60

61 Materials and Methods

62 Mice

63 hAPP-J20 mice were generated in-house and maintained on a C57BL/6J genetic background

64 (Mucke et al., 2000). Mouse pups were weaned 4-6 weeks after birth and housed up to 5 per

65 cage. Mice were fed a regular chow diet (PicoLab Rodent Diet 5053, TestDiet) and maintained

66 on a 12-hour light/dark cycle. All animal experiments were approved by the Institutional Animal 
67 Care and Use Committee at University of California-San Francisco under protocol AN105527-

6803.

69

Compounds and Reagents

71 All chemicals were purchased from Sigma. trans-ISRIB was provided by Dr. Peter Walter

72 (University of California-San Francisco). ISRIB was dissolved in DMSO, then diluted 100-fold

73 in sterile-filtered $0.9 \%$ saline $\mathrm{pH} 7.0$ to a final concentration of $15 \mu \mathrm{g} / \mathrm{mL}(0.25 \mathrm{mpk})$ or $6 \mu \mathrm{g} / \mathrm{mL}$

74 (0.1 mpk). For the $2.5 \mathrm{mpk}$ dose, ISRIB was dissolved in $1: 1 \mathrm{DMSO} / \mathrm{PEG}_{400}$ at $0.75 \mathrm{mg} / \mathrm{mL}$ and 75 administered at $3.3 \mu \mathrm{L} / \mathrm{g}$. Treatment solutions were prepared fresh daily from the appropriate 76 DMSO stock solution.

\section{Western blot analysis}

Mice were anesthetized with 2,2,2-Tribromoethanol (Avertin), perfused with $0.9 \%$ saline, and their hemibrains removed and snap frozen on dry ice and stored at $-80^{\circ} \mathrm{C}$. Prior to dissection, the hemibrains were thawed for 1 hour on ice, then dentate gyrus and cortex dissected and refrozen

82 on dry ice. Samples were then thawed and homogenized on a magnetic bead homogenizer

83 (NextAdvance Storm 24), followed by sonication (Episonic), in homogenization buffer (1x PBS, 1mM DTT, 0.5mM EDTA, 0.5\% Triton X-100, 0.1M PMSF, 1x HALT TM protease/phosphatase

85 inhibitor) at $4^{\circ} \mathrm{C}$. Homogenates were then centrifuged to pellet insoluble material, and the supernatants used for analysis. Protein concentrations were determined by the Bradford assay

87 (Bio-rad)(Bradford, 1976). Equal amounts of protein $(15 \mu \mathrm{g})$ prepared in 1x NuPAGE LDS sample buffer (Life Technologies) and 1x sample reducing buffer (Life Technologies) were 
89 loaded into the running lanes and electrophoresed at $180 \mathrm{~V}$ for 2 hours on $4-12 \%$ Bis-Tris (Life

90 Technologies) gels using a 1x MOPS buffer. Gels were soaked in 2x transfer buffer (Life

91 Technologies) $+10 \%$ methanol for 20 minutes, then proteins transferred to a nitrocellulose

92 membrane using a dry transfer system (iBlot, Life Technologies). Membranes were blocked

93 with either 5\% BSA in Tris-buffered saline (TBS) (eIF2 $\alpha$ ) or with Odyssey blocking buffer (LI-

94 COR) (ATF4) for 1 hour at room temp, then washed with TBS $+0.1 \%$ Tween 20 (TBST) three

95 times for 5 minutes each. Membranes were incubated with primary antibody in 5\% BSA-TBST

96 overnight at $4^{\circ} \mathrm{C}$, washed three times with TBST, then incubated with matching secondary

97 antibody conjugated to IRDye (LI-COR) in Odyssey blocking buffer (LI-COR) + 0.2\% Tween

9820 for 1 hour at room temp. Membranes were washed three times with TBST, then imaged on an

99 Odyssey CLx infrared imaging system (LI-COR).

Antibodies and dilutions used for western blotting

102

ATF4 (Santa Cruz) $\quad 1: 200$

103

GAPDH (Millipore)

$1: 1000$

104

IRDye $2^{\circ} \quad(\mathrm{LI}-\mathrm{COR})$

$1: 10,000$

105

106

Behavioral testing

107 Mice were group-housed during all behavioral tests. All control mice were littermate controls.

108 The investigator performing the behavioral testing was blinded to genotype and treatment. The

109 mice were handled prior to the start of behavioral testing. Five days prior to the start of the

110 experiment the experimenter placed his hand in the cage for 5 minutes. On the second through 
111 fourth days of handling the experimenter placed his hand in the cage for 1 minute, then

112 transferred the mice from the home cage to a clean cage for 2 minutes. On the day prior to the

113 start of the experiment the mice were transferred to the experimental room and allowed to

114 acclimate for 30 minutes, then handled for 1 minute and transferred from the home cage to a

115 clean cage for 2 minutes.

116

117

Morris water maze

118 The water maze consisted of a pool $(122 \mathrm{~cm}$ diameter $)$ filled with water opacified with nontoxic

119 white tempera paint powder, and surrounded by extramaze cues. For the pilot MWM \#1, mice

120 were trained to find a hidden platform $(14 \times 14 \mathrm{~cm}$, submerged $1 \mathrm{~cm})$ over 6 trials. For MWM \#2,

121 mice were trained over 11 trials. The platform location remained the same throughout hidden

122 platform training, but the drop location varied semi-randomly between trials. Each trial was 120

123 seconds in length, and was performed once per day except for the last two days for MWM \#2, in

124 which 2 trials were performed per day (trials 8 and 9, and 10 and 11 were performed on the same

125 day). The trial ended when the mouse found the platform, but the mouse was required to remain

126 on the platform for 10 seconds prior to removal from the pool. After removal from the pool, the

127 mouse was immediately injected with ISRIB by the intraperitoneal (i.p.) route, then placed back

128 into the home cage, as previously described (Sidrauski et al., 2013). Memory for the location of

129 the hidden platform was tested with 60 -second spatial probe trials performed 24 and 72 hours

130 after the final training trial. The drop location was $180^{\circ}$ from where the platform was placed

131 during hidden platform training. The same drop location was used for both spatial probe trials.

132 Swim paths were recorded and analyzed using an Ethovision XT video tracking system (Noldus

133 Information Technology). Swim speeds were not different between experimental groups. 
134 Training performance measures included latency to find the platform and distance traveled to

135 find the platform. Probe performance measures included percent time spent in the target

136 quadrant, latency to first cross the platform location, and the number of platform crossings.

\section{Contextual Fear Conditioning}

139 Mice were placed in near infrared fear conditioning chambers (Med Associates Inc.) that

140 contained distinct tactile, visual (variable light intensity set at 3 ), and olfactory (1\% acetic acid

141 solution) cues. The mice were allowed to explore the chamber for 2 minutes, at which time a

$1420.35 \mathrm{~mA}$ foot shock was delivered for 1 second. The activity of the mice was recorded for 1

143 minute post-shock, after which the mice were removed from the chambers and immediately

144 treated with ISRIB, as previously described (Sidrauski et al., 2013). Twenty-four hours later the 145 mice were placed back into the same chambers and movements were observed for 8 minutes.

146 Movements were recorded using high speed monochrome digital video cameras and analyzed

147 with VideoFreeze software (Med Associates Inc.). Chambers were cleaned with 70\% ethanol 148 between each testing session.

Statistical analysis

151 Statistical analysis was performed using Prism 6 (GraphPad Software) or the statistical

152 programming language R (http://www.R-project.org/). Normality was assessed using the D'Agostino \& Pearson omnibus test. Variance between groups was assessed using the $F$ test.

154 For comparisons of normal distributions with equal variances, a two-tailed unpaired $t$ test was 155 used. For comparisons of distributions in which one or both deviated from normality, a two- 
156 tailed Mann-Whitney test was used. Quadrant preference in the Morris water maze was assessed

157 using a two-tailed one-sample $t$ test against a theoretical mean of $25 \%$. Treatment effects in the

158 pilot MWM probe were analyzed using a one-way ANOVA. Treatment effects in the J20 MWM

159 probe were analyzed using a two-way ANOVA with Sidak's test for multiple comparisons.

160 Morris water maze training data were analyzed using a linear mixed effects regression model for

161 censored responses (Vaida et al., 2007) implemented in the R package lmec (Vaida and Liu).

162 Gender and ISRIB were included as trial modifiers. Gender was removed from the final model

163 after no significant effect was observed. Random mouse-level intercepts and slopes accounted

164 for the correlation among repeated observations. Goodness of fit was analyzed by inspection of

165 residuals. Differences were considered significant if the 5-95\% confidence interval did not cross

166 zero. Values reported are mean \pm standard error of the mean. Differences were considered

167 significant at $p<0.05$

$169 \underline{\text { Results }}$

A marker of ISRIB target engagement cannot be observed in brain homogenate from nontransgenic or hAPP-J20 mice

172 The transcription factor ATF4 has been used as a marker of target engagement in previously

173 published preclinical trials of ISRIB in disease models in which the level of this protein is

174 elevated (Halliday et al., 2015; Palam et al., 2015). In order to determine whether we could also

175 use levels of this protein as a marker of ISRIB target engagement in the brain, we analyzed brain

176 homogenates from two separate brain regions - cortex and dentate gyrus - in nontransgenic and

177 hAPP-J20 mice at three different ages: 2-3, 6-7, and 12-13 months. Consistent with previous 
178 studies, we were unable to detect protein levels of ATF4 in nontransgenic mice by western

179 blotting in either brain region (Fig. 1A) (Halliday et al., 2015). We were also unable to detect

180 ATF4 in hAPP-J20 mice in these same brain regions. We noted a very weak band at

181 approximately $50 \mathrm{kD}$ in the brain homogenates that appeared to be a nonspecific background

182 band, but we nevertheless quantified this band on the possibility that it represented a uniquely

183 post-translationally modified ATF4. The levels of this protein were not different between NTG

184 and J20 mice in either cortex or dentate gyrus in all 3 age groups (Fig. 1B; unpaired $t$ test, $p>$

185 0.05). These results suggest that the ISR is not significantly elevated in hAPP-J20 mice or in

186 nontransgenic mice. Therefore, we did not have a protein marker of ISRIB target engagement in

187 the brain for this preclinical drug study, similar to previous studies on memory enhancement

188 with ISRIB (Sidrauski et al., 2013; Di Prisco et al., 2014).

In a pilot behavioral experiment, ISRIB showed a trend towards enhancement of spatial memory

in the Morris water maze

192

193

194

195

196

197

198

199

200

Although we did not have a marker of target engagement in the brain for ISRIB, we decided to proceed with a pilot study to test its effects on learning and memory in nontransgenic mice. We used the Morris water maze as a test of spatial learning and memory (Morris, 1984). We treated mice with $0.1 \mathrm{mg} / \mathrm{kg}$ (mpk) or $0.25 \mathrm{mpk}$ ISRIB by intraperitoneal (i.p.) injection immediately after finishing each training session in the Morris water maze (MWM). We used a weak training protocol of one trial per day in order to increase our chances of observing an enhancement in learning and/or memory, as previously described (Sidrauski et al., 2013). Twenty-four and seventy-two hours after completion of the last training trial, we tested memory for the location of the hidden platform in probe trials. We did not observe a difference between control or ISRIB- 
201 treated mice at either dose in the time required to locate the hidden platform during training, or

202 the distance traveled before locating the hidden platform (linear mixed effects regression models,

203 non-significant 5-95\% confidence intervals) (Fig. 2A). In the 24-hour probe trial, we did not

204 observe a significant difference between control and ISRIB-treated mice in 3 separate outcome

205 measures: percent time spent in the target quadrant (one-way ANOVA, $F_{(2,42)}=0.45, p=0.64$ ),

206 latency to first cross the platform location (one-way ANOVA, $F_{(2,39)}=1.18, p=0.32$ ), or the

207 number of platform crossings (one-way ANOVA, $F_{(2,42)}=0.93, p=0.40$ ) (Fig. 2B). However,

208 there was a dose-related numerical trend towards improved performance in the latency measure

209 with ISRIB treatment. At 72 hours, the mice treated with $0.25 \mathrm{mpk}$ ISRIB spent significantly

210 more time in the target quadrant compared to chance, whereas the control and $0.1 \mathrm{mpk}$ ISRIB-

211 treated mice did not (one sample $t$ test against 25\%: vehicle $p=0.26$, ISRIB $0.1 \mathrm{mpk} p=0.85$,

212 ISRIB $0.25 \mathrm{mpk} p=0.009$ ) (Fig. 2C). There was also a general numerical trend towards

213 improvement in all three memory outcome measures with $0.25 \mathrm{mpk}$ ISRIB compared to control

214 (Fig. 2C). We verified drug exposure on day 6 of the MWM by injecting 2 satellite mice with

$2150.25 \mathrm{mpk}$ ISRIB and measuring plasma levels 2.5 hours post-injection. Both satellite mice

216 showed plasma levels above the $\mathrm{IC}_{50}(5 \mathrm{nM})$ for ISRIB (Table 1) (Sidrauski et al., 2013). ISRIB

217 is not significantly excluded from the brain by the blood-brain-barrier, so plasma ISRIB levels

218 roughly approximate compound levels in the brain (Sidrauski et al., 2013; Halliday et al., 2015).

219 Thus, while we did not observe a benefit in training measures with ISRIB treatment in this pilot

220 study, a number of outcome measures in the probe trials suggested a general statistically

221 insignificant trend towards improvement in long-term memory with ISRIB treatment, especially

222 at the $0.25 \mathrm{mpk}$ dose. 
225 Given our observations in the Morris water maze probe trials, we decided to use the same mice

226 to test whether contextual fear memory, which is largely dependent upon the amygdala and

227 hippocampus, could be enhanced with ISRIB treatment. For this assay, we used a different

228 vehicle formulation for ISRIB in order to increase the amount of compound that could be

229 delivered by the i.p. route of administration. We found that a minimal volume of $50 \% \mathrm{DMSO} /$

$23050 \%$ polyethyleneglycol (PEG) was sufficient to dissolve and deliver the hydrophobic ISRIB

231 compound at a 10-fold greater dose (2.5 mpk) than what could be achieved with $1 \%$

232 DMSO/saline during the pilot MWM experiment. Nine days after completion of the MWM 72-

$233 \mathrm{hr}$ probe trial, mice were placed in fear conditioning chambers and observed for freezing

234 behavior prior to and after delivery of a mild electrical foot shock. Prior to receiving the shock,

235 the control and planned treatment group both showed very low freezing behavior. After delivery

236 of the shock, both groups demonstrated increased freezing behavior, with no significant

237 difference between control and planned treatment groups (Mann-Whitney test, $p=0.18$ ) (Fig.

238 3A). After removal from the chambers, the mice were treated with $2.5 \mathrm{mpk}$ ISRIB with a single

239 i.p. injection and placed back into their home cages. Twenty-four hours later, the mice were

240 placed into the chamber (context) and their freezing behavior quantified. Over the first 4

241 minutes, the second 4 minutes, or the total 8 minutes of observation, we did not observe a

242 significant difference in freezing behavior between vehicle- and ISRIB-treated groups (first 4

243 minutes unpaired $t$ test, $p=0.38$; second 4 minutes Mann-Whitney test, $p=0.24$; total 8 minutes

244 Mann-Whitney test, $p=0.31$ ). (Fig. 3B). Thus, we did not observe an enhancement of fear

245 memory with ISRIB using this testing paradigm. 
247 ISRIB failed to rescue spatial learning and memory deficits in hAPP-J20 mice

248 Given the potential trend toward improvement in memory in the MWM with ISRIB at $0.25 \mathrm{mpk}$, 249 and the ability to deliver greater amounts of the compound using the DMSO/PEG vehicle, we 250 proceeded to test whether ISRIB might improve learning and memory in the hAPP-J20 (J20)

251 model of Alzheimer's disease. A cohort consisting of nontransgenic (NTG) and J20 mice aged

252 6-7 months were used to test enhancement of spatial learning and memory in the MWM with two 253 separate doses of ISRIB: $0.25 \mathrm{mpk}$ in 1\% DMSO/saline, and $2.5 \mathrm{mpk}$ in DMSO/PEG. Because

254 we were unsure how the DMSO/PEG vehicle used to deliver the higher ISRIB dose may affect

255 the mice, we controlled for both vehicle formulations. Each control and treatment group

256 consisted of approximately $12-15$ mice. To increase memory for the location of the platform

257 compared to the pilot MWM study, we performed 5 additional training sessions, with 2 trials per

258 day on the last 2 days. Mice were treated with vehicle (1\% DMSO/saline or DMSO/PEG) or

259 ISRIB at $0.25 \mathrm{mpk}$ or $2.5 \mathrm{mpk}$ i.p. immediately after completion of each training session. Probe

260 trials were performed at 24 and 72 hours after the last training session to test spatial memory.

261 While we observed a significant learning deficit in J20 compared to NTG mice (Supplementary

262 Fig. 1), we did not observe an improvement in learning during the training trials with ISRIB

263 treatment at either dose in NTG or J20 mice (linear mixed effects regression models, non-

264 significant 5-95\% confidence intervals) (Fig. 4A-D). We also did not observe an improvement

265 in 3 separate measures of spatial memory during the 24 hour probe trial in NTG or J20 mice at

266 either dose (Fig. 4E-G; see figure legend for statistical details). When we tested the mice again

267 at 72 hours in the probe trial (Fig. 4H-J), we did not observe significantly positive treatment

268 effects (see figure legend for statistical details). To increase our power to detect an ISRIB

269 treatment effect, we pooled the vehicle control groups in NTG and J20 mice and reanalyzed the 
270 data (Supplementary Fig. 2). After pooling control groups, we did not detect an ISRIB treatment

271 effect (24 hour time in target quadrant two-way ANOVA: treatment $F_{(2,87)}=0.42, p=0.66$;

272 genotype $F_{(1,87)}=25.10, p<0.0001$; treatment $\mathrm{x}$ genotype $F_{(2,87)}=0.06, p=0.95$. 72 hour time

273 in target quadrant two-way ANOVA: treatment $F_{(2,87)}=0.61, p=0.55$; genotype $F_{(1,87)}=11.15$,

$274 p=0.001$; treatment $\mathrm{x}$ genotype $F_{(2,87)}=1.73, p=0.18$. See Supplementary Fig. 2 for additional

275 outcome measures). To verify that we had delivered the ISRIB compound to the mice, we tested

276 plasma levels of ISRIB in a satellite cohort of mice 2.5 hours after i.p. injection during trial 7.

277 Plasma ISRIB levels were above the $\mathrm{IC}_{50}(5 \mathrm{nM})$ in all mice (Table 1). We also tested whether

278 ISRIB was chemically stable in our DMSO stock solution used to prepare the treatment solutions

279 during MWM testing. Liquid chromatography-mass spectrometry (LC-MS) analysis of the stock

280 solution at $t=0$ and $t=3$ weeks showed no change in retention time or mass of the ISRIB

281 compound (data not shown, available upon request). Thus, ISRIB did not reverse spatial

282 learning or memory deficits in J20, nor did it improve memory in NTG mice in this MWM

283 experiment despite adequate drug exposure.

\section{$\underline{\text { Discussion }}$}

286

287

In this preclinical drug study we tested whether ISRIB would rescue the learning and memory deficits observed in the J20 mouse model of Alzheimer's disease. We also tested whether it would enhance spatial learning and memory, as well as fear memory, in nontransgenic mice. We did not observe a significant rescue of spatial learning or memory in 6-7 month-old J20 mice with ISRIB. In nontransgenic mice, we observed a general trend towards enhanced long-term memory at the $0.25 \mathrm{mpk}$ dose in a pilot MWM cohort, but we were unable to repeat this finding in a second cohort. We also did not find an enhancement of fear memory at 24 hours in 6-7 
293 month-old nontransgenic mice. Although we did not have a marker of target engagement for

294 these experiments, similar to previous studies on memory enhancement with ISRIB (Sidrauski et

295 al., 2013; Di Prisco et al., 2014), we measured plasma levels of ISRIB in two satellite cohorts of

296 mice during the MWM experiments and verified excellent drug exposure at both the $0.25 \mathrm{mpk}$

297 and $2.5 \mathrm{mpk}$ doses. ISRIB readily crosses the blood-brain-barrier (Sidrauski et al., 2013;

298 Halliday et al., 2015).

299 ISRIB has been shown to extend survival in disease models that show elevated ISR activity, such 300 as prion disease mouse models (Halliday et al., 2015). In a prion disease model, treatment with 301 ISRIB reduced inhibition of protein translation as measured by the levels of ATF4, a marker of 302 ISR activity, and extended survival by approximately 1 week. This beneficial effect on survival 303 was achieved by chronic daily i.p. administration of $0.25 \mathrm{mpk}$ ISRIB over the course of five to 304 seven weeks (Halliday et al., 2015). In contrast, ISRIB's effects on learning and memory have 305 been demonstrated after a single i.p. injection in wildtype rodents (Sidrauski et al., 2013; Di 306 Prisco et al., 2014), suggesting that chronic administration of ISRIB is not required for cognitive 307 enhancement, nor presumably for rescue of cognitive deficits via the same mechanism, at least in 308 disease models where the ISR is not elevated such as in the J20 mouse model. Both the beneficial survival effect in prion-infected mice and the beneficial effects on cognition in NTG mice with ISRIB treatment are thought to relate to the compound's effects on protein translation. It is possible that chronic dosing of ISRIB, most easily performed through a food formulation,

312 would also lead to an improvement in learning and memory. This would be an interesting future 313 direction for research on ISRIB. Given that protein translation rates are highly regulated, it 314 seems quite possible that increasing total protein translation throughout an organism for an 315 extended period of time could in fact be detrimental to cognition, either directly or through a 
316 secondary toxicity mechanism. It is also unclear whether chronic ISRIB administration would

317 allow homeostatic compensation to occur in the protein translation system prior to the learning

318 test, thereby preventing the desired enhancement on learning and memory. Answering such

319 questions would be highly facilitated by a marker of ISRIB target engagement that is directly

320 related to the underlying mechanism(s) by which it is thought to enhance cognition. One such

321 mechanism that has been proposed is modulation of long term depression (LTD) (Di Prisco et

322 al., 2014). Object-place learning has been shown to be dependent upon proper induction of

323 hippocampal LTD, which appears to require a transcriptional program that is induced by eIF $2 \alpha$

324 phosphorylation (Di Prisco et al., 2014). Acute treatment with ISRIB interfered with proper

325 object-place learning by preventing activation of this transcriptional program and induction of

326 LTD (Di Prisco et al., 2014). Interestingly, A $\beta$ has been shown to cause elevated LTD in rodent

327 hippocampal slice culture (Shankar et al., 2008; Luscher and Huber, 2010; Pozueta et al., 2013),

328 which may be one mechanism by which hAPP overexpression contributes to learning and

329 memory deficits observed in AD mouse models. Performance in these behavioral assays can

330 also be influenced by non-neuronal cell types such as astrocytes (Orr et al., 2015), which are

331 pathologically activated in the presence of $A \beta$ plaques (Serrano-Pozo et al., 2016) and are also

332 likely affected by treatment with ISRIB. Cell-type specific biochemical markers, rather than

333 electrophysiologic markers, of target engagement that relate to performance in these behavioral

334 tasks would be helpful for the study of ISRIB's effects on cognition in wildtype and disease

335 models.

336 In addition to the lack of effects observed in the J20 model, we were unable to replicate the

337 memory-enhancing effects with ISRIB treatment in nontransgenic mice. There are a number of

338 possibilities that might explain why we were unable to see an effect on memory in nontransgenic 
339 mice. The first possibility is that our training paradigm in the MWM was not weak enough to

340 allow a benefit to emerge in ISRIB-treated mice. While this possibility may explain our inability

341 to see an effect during training in the pilot MWM \#1 and MWM \#2 given that all NTG mice

342 learned the location of the hidden platform at equivalent rates, it is unlikely to explain our

343 inability to see an effect in the probe trials, as the control mice in MWM \#1 showed rather weak

344 memory for the location of the platform at 24 and 72 hours. When we increased training in

345 MWM \#2 with an additional 5 trials, we noted improved memory for the location of the platform

346 in NTG mice at 24 and 72 hours compared to mice tested in MWM \#1, as expected. While we

347 again noted a general trend towards improvement in latency to target at the $0.25 \mathrm{mpk}$ dose

348 similar to the observation in the pilot MWM \#1, we did not find a significant improvement in

349 memory in NTG mice in MWM \#2. Therefore, while a weaker training protocol may make it

350 more likely for differences to be observed in learning rates with ISRIB treatment, it is unlikely to

351 lead to better separation between groups in the probe trial. A second possibility is that our study

352 was insufficiently powered to see a beneficial effect on learning and memory in NTG mice.

353 Given the general trends observed in MWM \#1 and the relatively small effect size, a study that is

354 much more highly powered might be able to show significance. We note that each experimental

355 group in this study contained approximately 12-15 mice, for a total of $>100$ mice (including

356 satellite mice) for MWM \#2. The size of each experimental group is equivalent to or larger than

357 those used in previous behavioral studies with ISRIB (Sidrauski et al., 2013; Di Prisco et al.,

358 2014). To increase power in a future MWM experiment, the number of treatment groups would

359 need to be reduced, and/or separate experiments would need to be analyzed in a pooled manner.

360 A third (and related) possibility is that the MWM is a relatively insensitive test of spatial learning

361 and memory. Newer behavioral assays of spatial learning and memory, such as the interactive 
362 place avoidance test, might better detect an enhancement of learning and memory with ISRIB

363 treatment (Cimadevilla et al., 2001). A final possibility is that the memory-enhancing effect of

364 ISRIB is age-dependent. In our study we tested 6-7 month-old mice, whereas the prior study that

365 demonstrated memory enhancement with ISRIB used 2 month-old mice.

366 One potential reason why we were unable to observe an enhancement of fear memory with

367 ISRIB may have been the sequencing of the behavioral tests performed. Contextual fear

368 conditioning was performed in the same cohort of mice after they had been exposed to the

369 MWM. This may have explained their rather low baseline freezing rates prior to receiving a foot

370 shock, as desensitization may have occurred with prior exposure to the pool of water in the

371 MWM. Future tests of fear memory enhancement with ISRIB treatment would likely benefit

372 from using a naïve cohort of mice.

373 In conclusion, we did not find a significant beneficial effect of ISRIB treatment in hAPP-J20

374 mice or NTG mice. Future preclinical studies with ISRIB on memory enhancement in wildtype

375 animals and disease models would benefit from robust markers of target engagement in the brain

376 that are directly related to the molecular mechanism(s) by which memory enhancement is

377 thought to occur.

378

379 Acknowledgments

380 The authors thank Gui-qiu Yu, Xin Wang, and Wei-kun Guo for mouse colony maintenance,

381 Shaun Fontaine for performing LC-MS analysis, and Mariel Finucane for advice on statistical

382 model construction and analysis. The authors thank Carmela Sidrauski, Pascal Sanchez, Kaitlyn

383 Ho, and the Gladstone Behavioral Core staff for helpful discussions, and Lennart Mucke for 
384 reviewing the manuscript. The authors also thank Monica Dela Cruz, Amy Cheung, and 385 Courtney Dickerson for administrative assistance.

\section{References}

388 Alzheimer's Association (2015) 2015 Alzheimer's disease facts and figures. Alzheimers Dement 389 $11: 332-384$.

Bradford MM (1976) A rapid and sensitive method for the quantitation of microgram quantities of protein utilizing the principle of protein-dye binding. Anal Biochem 72:248-254.

Cimadevilla JM, Fenton AA, Bures J (2001) New spatial cognition tests for mice: passive place avoidance on stable and active place avoidance on rotating arenas. Brain Res Bull 54:559-563.

Cisse M, Halabisky B, Harris J, Devidze N, Dubal DB, Sun B, Orr A, Lotz G, Kim DH, Hamto P, Ho K, Yu GQ, Mucke L (2011) Reversing EphB2 depletion rescues cognitive functions in Alzheimer model. Nature 469:47-52.

Di Prisco GV, Huang W, Buffington SA, Hsu CC, Bonnen PE, Placzek AN, Sidrauski C, synaptic plasticity and memory. Cell 129:195-206. Neurosci 17:1073-1082. 
406 Halliday M, Mallucci GR (2015) Review: Modulating the unfolded protein response to prevent

407

408

409

410

411

412

413

414

415

416

417

418

419

420

421

422

423

424

425

426

427

428

neurodegeneration and enhance memory. Neuropathol Appl Neurobiol 41:414-427.

Halliday M, Radford H, Sekine Y, Moreno J, Verity N, le Quesne J, Ortori CA, Barrett DA, Fromont C, Fischer PM, Harding HP, Ron D, Mallucci GR (2015) Partial restoration of protein synthesis rates by the small molecule ISRIB prevents neurodegeneration without pancreatic toxicity. Cell Death Dis 6:e1672.

Kida S, Serita T (2014) Functional roles of CREB as a positive regulator in the formation and enhancement of memory. Brain Res Bull 105:17-24.

Luscher C, Huber KM (2010) Group 1 mGluR-dependent synaptic long-term depression: mechanisms and implications for circuitry and disease. Neuron 65:445-459.

Morris R (1984) Developments of a Water-Maze Procedure for Studying Spatial-Learning in the Rat. J Neurosci Meth 11:47-60.

Mucke L, Masliah E, Yu GQ, Mallory M, Rockenstein EM, Tatsuno G, Hu K, Kholodenko D, Johnson-Wood K, McConlogue L (2000) High-level neuronal expression of abeta 1-42 in wild-type human amyloid protein precursor transgenic mice: synaptotoxicity without plaque formation. J Neurosci 20:4050-4058.

Orr AG, Hsiao EC, Wang MM, Ho K, Kim DH, Wang X, Guo W, Kang J, Yu GQ, Adame A, Devidze N, Dubal DB, Masliah E, Conklin BR, Mucke L (2015) Astrocytic adenosine receptor A2A and Gs-coupled signaling regulate memory. Nat Neurosci 18:423-434.

Palam LR, Gore J, Craven KE, Wilson JL, Korc M (2015) Integrated stress response is critical for gemcitabine resistance in pancreatic ductal adenocarcinoma. Cell Death Dis 6:e1913.

Pozueta J, Lefort R, Shelanski ML (2013) Synaptic changes in Alzheimer's disease and its models. Neuroscience 251:51-65. 
429 Roberson ED, Scearce-Levie K, Palop JJ, Yan F, Cheng IH, Wu T, Gerstein H, Yu GQ, Mucke

430 L (2007) Reducing endogenous tau ameliorates amyloid beta-induced deficits in an

431

432

433

434

435

436

437

438

439

440

441

442

443

444

445

446

447

448

449

450

451

Alzheimer's disease mouse model. Science 316:750-754.

Sanchez-Mejia RO, Newman JW, Toh S, Yu GQ, Zhou Y, Halabisky B, Cisse M, Scearce-Levie K, Cheng IH, Gan L, Palop JJ, Bonventre JV, Mucke L (2008) Phospholipase A2 reduction ameliorates cognitive deficits in a mouse model of Alzheimer's disease. Nat Neurosci 11:1311-1318.

Sanchez PE, Zhu L, Verret L, Vossel KA, Orr AG, Cirrito JR, Devidze N, Ho K, Yu GQ, Palop JJ, Mucke L (2012) Levetiracetam suppresses neuronal network dysfunction and reverses synaptic and cognitive deficits in an Alzheimer's disease model. Proceedings of the National Academy of Sciences of the United States of America 109:E2895-2903.

Sekine Y, Zyryanova A, Crespillo-Casado A, Fischer PM, Harding HP, Ron D (2015) Stress responses. Mutations in a translation initiation factor identify the target of a memoryenhancing compound. Science 348:1027-1030.

Serrano-Pozo A, Betensky RA, Frosch MP, Hyman BT (2016) Plaque-Associated Local Toxicity Increases over the Clinical Course of Alzheimer Disease. Am J Pathol 186:375-384.

Shankar GM, Li S, Mehta TH, Garcia-Munoz A, Shepardson NE, Smith I, Brett FM, Farrell MA, Rowan MJ, Lemere CA, Regan CM, Walsh DM, Sabatini BL, Selkoe DJ (2008) Amyloid-beta protein dimers isolated directly from Alzheimer's brains impair synaptic plasticity and memory. Nat Med 14:837-842.

Sidrauski C, McGeachy AM, Ingolia NT, Walter P (2015a) The small molecule ISRIB reverses the effects of eIF2alpha phosphorylation on translation and stress granule assembly. Elife 4. 
452 Sidrauski C, Tsai JC, Kampmann M, Hearn BR, Vedantham P, Jaishankar P, Sokabe M, Mendez

453

454

455

456

457

458

459

460

461

462

463

464

465

466

467

468

469

470

471

472

AS, Newton BW, Tang EL, Verschueren E, Johnson JR, Krogan NJ, Fraser CS,

Weissman JS, Renslo AR, Walter P (2015b) Pharmacological dimerization and activation

of the exchange factor eIF2B antagonizes the integrated stress response. Elife 4:e07314.

Sidrauski C, Acosta-Alvear D, Khoutorsky A, Vedantham P, Hearn BR, Li H, Gamache K, Gallagher CM, Ang KK, Wilson C, Okreglak V, Ashkenazi A, Hann B, Nader K, Arkin MR, Renslo AR, Sonenberg N, Walter P (2013) Pharmacological brake-release of mRNA translation enhances cognitive memory. Elife 2:e0498.

Stern E, Chinnakkaruppan A, David O, Sonenberg N, Rosenblum K (2013) Blocking the eIF2alpha kinase (PKR) enhances positive and negative forms of cortex-dependent taste memory. J Neurosci 33:2517-2525.

Vaida F, Liu L lmec: Linear Mixed-Effects Models with Censored Responses. In, R package version 1.0 Edition.

Vaida F, Fitzgerald AP, DeGruttola V (2007) Efficient hybrid EM for linear and nonlinear mixed effects models with censored response. Comput Stat Data An 51:5718-5730.

Walter P, Ron D (2011) The unfolded protein response: from stress pathway to homeostatic regulation. Science 334:1081-1086.

Webster SJ, Bachstetter AD, Nelson PT, Schmitt FA, Van Eldik LJ (2014) Using mice to model Alzheimer's dementia: an overview of the clinical disease and the preclinical behavioral changes in 10 mouse models. Front Genet 5:88. 


\section{Table $\mathbf{1}$ (on next page)}

ISRIB pharmacokinetic data.

$\mathrm{mpk}=\mathrm{mg} / \mathrm{kg}$. The IC $\mathrm{I}_{50}$ of ISRIB is $5 \mathrm{nM}$. ISRIB demonstrates excellent blood-brain-barrier penetration (Sidrauski et al., 2013; Halliday et al., 2015). 


\begin{tabular}{|c|c|c|c|c|c|}
\hline Experiment & Mouse & ISRIB Treatment (Vehicle) & $\begin{array}{l}\text { Time Post- } \\
\text { Administration }\end{array}$ & $\begin{array}{l}\text { Plasma } \\
\text { Concentration } \\
\text { (ng/mL) }\end{array}$ & $\begin{array}{l}\text { Plasma } \\
\text { Concentration } \\
\text { (nM) }\end{array}$ \\
\hline \multirow[t]{2}{*}{ Morris water maze \#1 } & 1 & 0.25 mpk (1\% DMSO/saline) & 2.5 hours & 65.3 & 145 \\
\hline & 2 & $0.25 \mathrm{mpk}$ (1\% DMSO/saline) & 2.5 hours & 71.9 & 159 \\
\hline \multirow[t]{5}{*}{ Morris water maze \#2 } & 1 & $0.25 \mathrm{mpk}$ (1\% DMSO/saline) & 2.5 hours & 66.6 & 148 \\
\hline & 2 & 0.25 mpk (1\% DMSO/saline) & 2.5 hours & 65.6 & 145 \\
\hline & 3 & $2.5 \mathrm{mpk}$ (DMSO/PEG) & 2.5 hours & 657 & 1456 \\
\hline & 4 & 2.5 mpk (DMSO/PEG) & 2.5 hours & 348 & 771 \\
\hline & 5 & 2.5 mpk (DMSO/PEG) & 2.5 hours & 286 & 634 \\
\hline
\end{tabular}




\section{Figure 1 (on next page)}

ATF4 as a pharmacodynamic marker in nontransgenic and hAPP-J20 mice.

(A) Cortex and dentate gyrus brain homogenates from hAPP-J20 (J20) and nontransgenic (NTG) mice at 2-3, 6-7, and 12-13 months (mo) of age were analyzed for ATF4 protein levels by western blotting ( $N=6$ per group). A representative blot is shown. While low levels of ATF4 could be observed in the untreated control 293T cell lysate, and elevated levels could be observed in the 293T cells treated with tunicamycin ( $\mathrm{Tm}$ ) and thapsigargin $(\mathrm{Tg})$ to induce ER stress, ATF4 could not be observed in NTG or J20 brain homogenate. (B) A weak band at $\sim 50 \mathrm{kD}$ in the brain homogenate samples was quantified on the possibility that this represented a post-translationally modified ATF4 unique to brain tissue. No differences in protein levels represented by this band were observed between NTG and 120 mice (unpaired $t$ test, $p>0.05)$. 

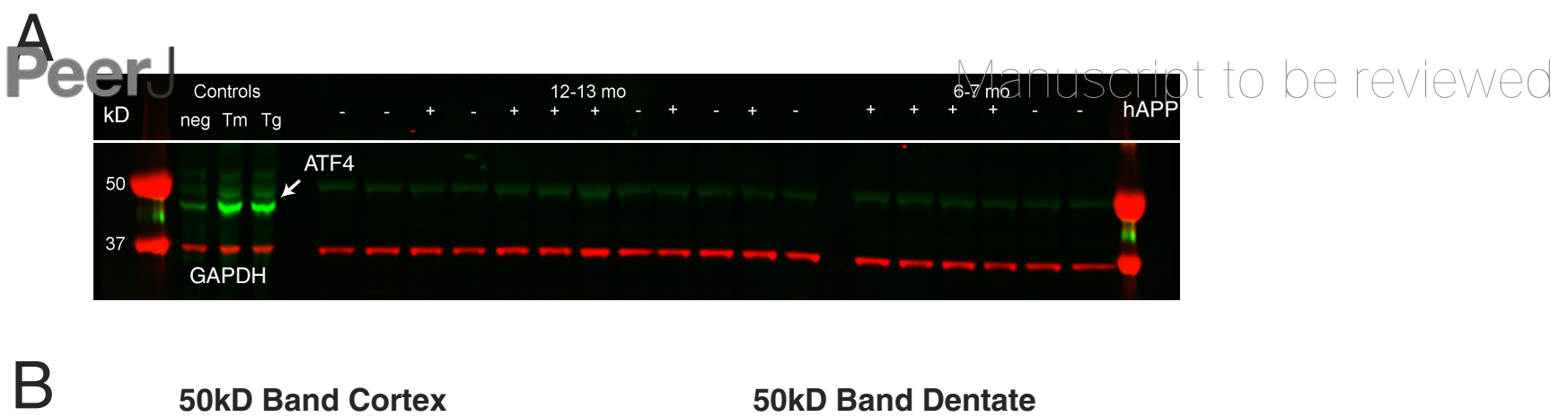

50kD Band Cortex

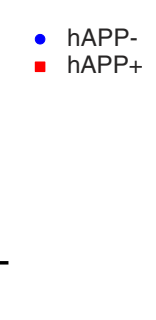

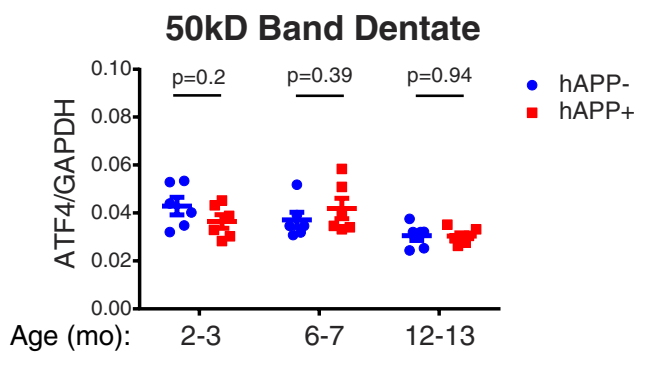




\section{Figure 2 (on next page)}

A pilot Morris water maze experiment suggests a trend towards enhanced long-term spatial memory with ISRIB treatment.

(A-C) 6-7 month-old nontransgenic mice were tested in the Morris water maze (MWM) $(\mathrm{N}=$ 15 per group). (A) MWM training. Mice were treated with vehicle (1\% DMSO/0.9\% saline), 0.1 $\mathrm{mg} / \mathrm{kg}$ (mpk) ISRIB, or $0.25 \mathrm{mpk}$ ISRIB ( $\mathrm{N}=15$ per group) immediately after completion of a MWM training session (1 trial per day). No differences in learning rates to find the hidden platform, in either latency or distance measures, were observed between mice treated with vehicle or ISRIB (linear mixed effects regression model, latency Trial*ISRIB $0.1 \mathrm{mpk}=-1.2$ sec/day $(5-95 \% \mathrm{Cl}=-10.5$ to $8.1 \mathrm{sec} /$ day), latency Trial*ISRIB $0.25 \mathrm{mpk}=-0.9 \mathrm{sec} /$ day $(5-$ $95 \% \mathrm{Cl}=-10.1$ to $8.3 \mathrm{sec} /$ day), distance Trial*ISRIB $0.1 \mathrm{mpk}=-35 \mathrm{~cm} /$ day $(5-95 \% \mathrm{Cl}=-207$ to $136 \mathrm{~cm} /$ day), distance Trial*ISRIB $0.25 \mathrm{mpk}=-36 \mathrm{~cm} /$ day $(5-95 \% \mathrm{Cl}=-207$ to 135 $\mathrm{cm} /$ day)). (B) Mice were tested in a probe trial 24 hours after completion of the last training trial. No significant improvement in memory for the location of the hidden platform was observed in multiple outcome measures, including percentage of time spent in the target quadrant (one sample $t$ test against 25\% (random chance, dotted line): vehicle $p=0.03$, ISRIB $0.1 \mathrm{mpk} p=0.23$, ISRIB $0.25 \mathrm{mpk} p=0.09$; one-way ANOVA, $F_{(2,42)}=0.45, p=0.64$ ) latency to first cross the target platform location (one-way ANOVA, $F_{(2,39)}=1.18, p=0.32$ ), and the number of platform crossings (one-way ANOVA, $F_{(2,42)}=0.93, p=0.40$ ), although numerically the values trended in the expected direction in the latency to target and crossing frequency measures in the $0.25 \mathrm{mpk}$ ISRIB group. (C) Mice were tested in a probe trial 72 hours after completion of the last training trial. Mice treated with $0.25 \mathrm{mpk}$ ISRIB spent more time in the target quadrant compared to chance (one sample $t$ test against 25\%: vehicle $p=$ 0.26 , ISRIB $0.1 \mathrm{mpk} p=0.85$, ISRIB $0.25 \mathrm{mpk} p=0.009$; one-way ANOVA, $F_{(2,42)}=2.57, p=$ $0.09)$. There was no significant improvement in latency to target (one-way ANOVA, $F_{(2,32)}=$ $1.50, p=0.24$ ) or crossing frequency (one-way ANOVA, $F_{(2,42)}=0.38, p=0.69$ ) outcome

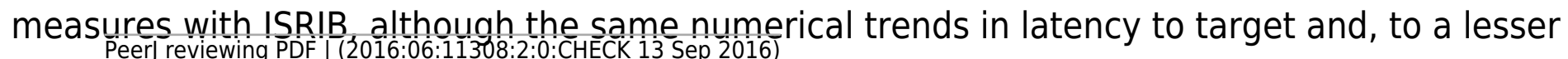


extent, crossing frequency were observed as in the 24-hour probe trial. ${ }^{*} p<0.05$, ${ }^{* *} p<$ 0.01 . 

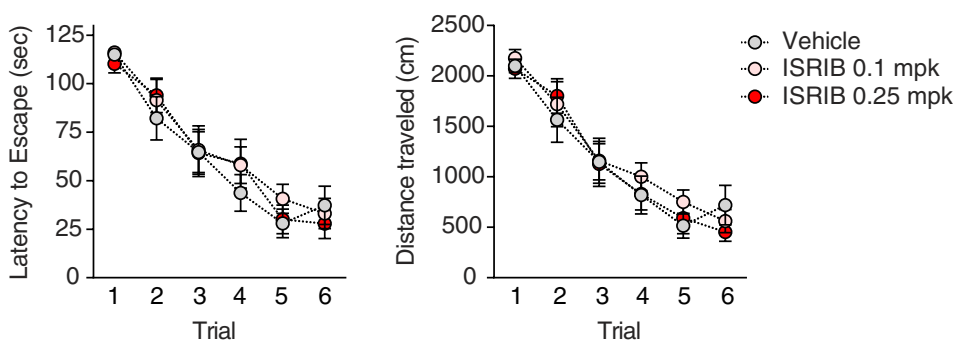

B

$24 \mathrm{hr} \%$ Target Quadrant

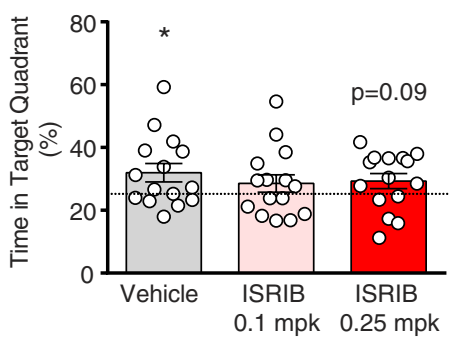

$24 \mathrm{hr}$ Latency to Target

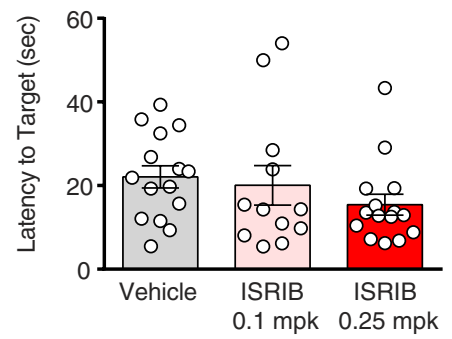

$\mathrm{C}$

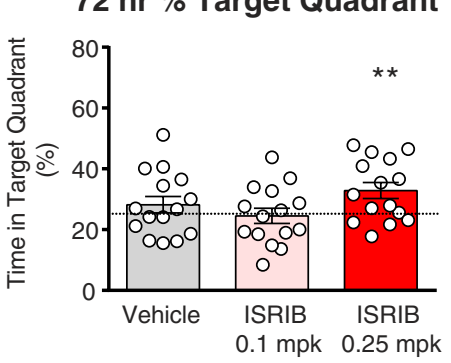

$72 \mathrm{hr}$ Latency to Target

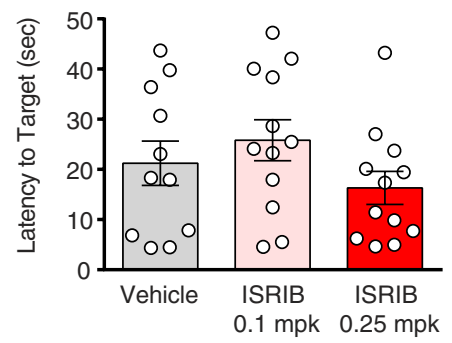

24hr Quadrant Occupancy

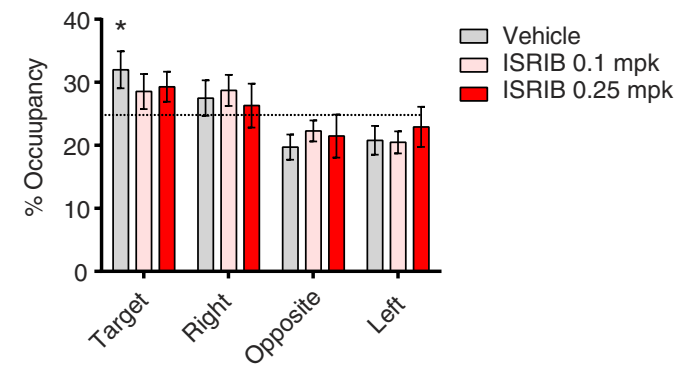

$24 \mathrm{hr}$ Crossing Frequency

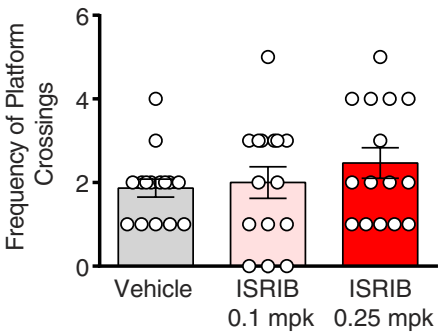

72hr Quadrant Occupancy

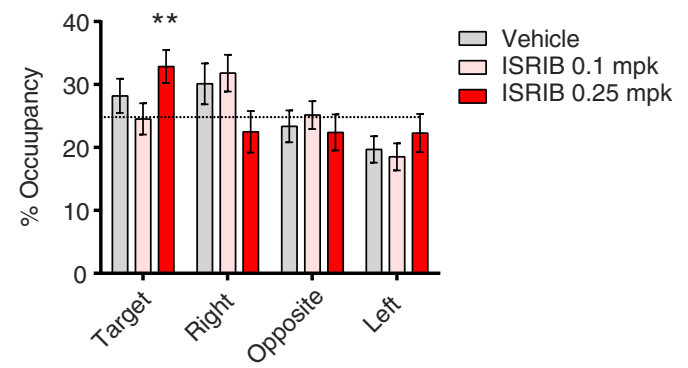

$72 \mathrm{hr}$ Crossing Frequency

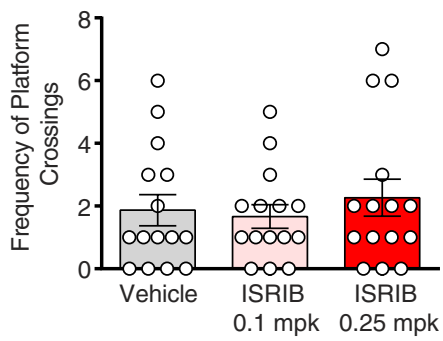




\section{Figure 3 (on next page)}

ISRIB does not show a beneficial effect on fear memory in a contextual fear conditioning paradigm.

(A, B) Mice that had been tested previously in the MWM were tested in fear conditioning. (A) Mice were placed in the chamber and allowed to explore for 2 minutes prior to delivery of a mild foot shock, after which their freezing behavior was quantified for 1 minute. They were then removed from the chamber and immediately treated with either vehicle (1:1 DMSO:PEG $\left.{ }_{400}, \mathrm{~N}=15\right)$ or $2.5 \mathrm{mpk}$ ISRIB $(\mathrm{N}=30)$. The mice showed no differences in freezing rates between treatment groups prior to and after receiving the foot shock (Mann-Whitney test). (B) 24 hours later the mice were placed back in the same chamber (context) and their freezing behavior was quantified over the course of 8 minutes. No difference in the amount of freezing was observed between vehicle- and ISRIB-treated groups during the first 4 minutes, the second 4 minutes, or the total 8 minutes of observation (first 4 minutes unpaired $t$ test, second 4 minutes Mann-Whitney test, total 8 minutes Mann-Whitney test). 
A

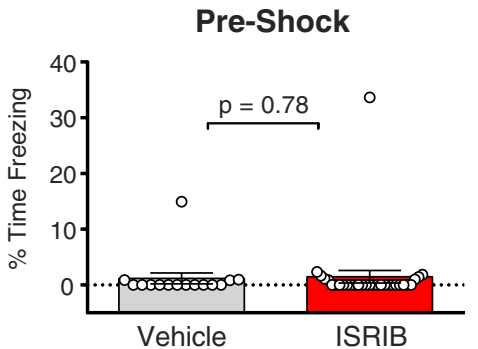

B

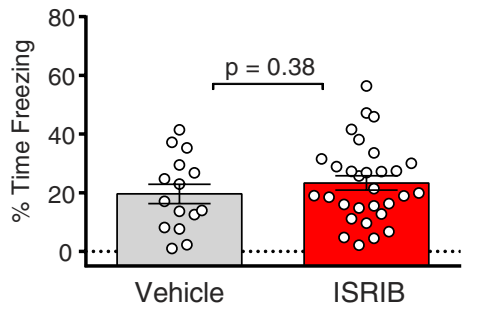

\section{Post-Shock}

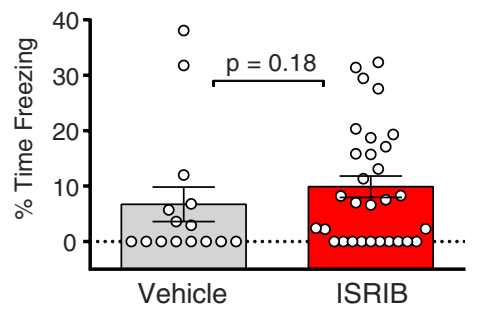

Context - 2nd 4 min

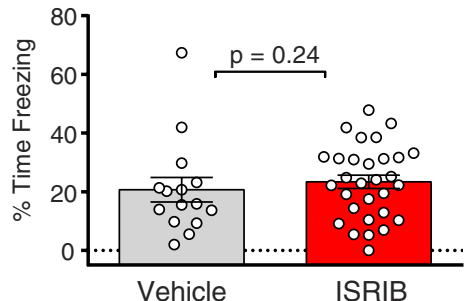

Context - Total 8 min

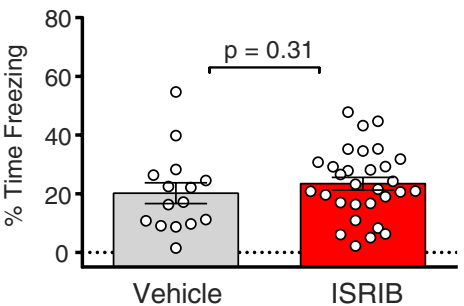




\section{Figure 4 (on next page)}

ISRIB does not rescue spatial learning and memory deficits in hAPP-J20 mice in the Morris water maze.

(A-J) A separate cohort of 6-7 month-old nontransgenic (NTG) and hAPP-J20 (J20) mice treated with either vehicle or ISRIB were tested in the MWM. (A-D) Mice were trained in the MWM over 11 trials. Mice were treated with vehicle (1\% DMSO/0.9\% saline or 50\% DMSO/50\% PEG) or ISRIB (0.25 mpk or $2.5 \mathrm{mpk}$ ) immediately after each training session. On days 8 and 9 , mice received two training sessions per day, and were injected immediately after the second session. (A) NTG mice were treated with vehicle (1\% DMSO/saline, $\mathrm{N}=12)$ or ISRIB $(0.25$ mpk in $1 \% \mathrm{DMSO} / \mathrm{saline}, \mathrm{N}=12$ ). (B) NTG mice were treated with vehicle (50\% DMSO/50\% PEG, $N=12$ ) or ISRIB (2.5 mpk in 50\% DMSO/50\% PEG, $N=12$ ). (C) $J 20$ mice were treated with vehicle (1\% DMSO/saline, $\mathrm{N}=11$ ) or ISRIB (0.25 mpk in $1 \% \mathrm{DMSO} / \mathrm{saline}, \mathrm{N}=12)$. (D) J20 mice were treated with vehicle (50\% DMSO/50\% PEG, $\mathrm{N}=10$ ) or ISRIB (2.5 mpk in 50\% DMSO/50\% PEG, $\mathrm{N}=12$ ). (Insets) Linear mixed effects regression models were used to analyze differences in learning rates between treatment and vehicle control groups. No significant differences in learning rates were observed in NTG mice or 320 mice with ISRIB treatment at either dose (linear mixed effects regression model, latency NTG Trial*ISRIB 0.25 $\mathrm{mpk}=-0.1 \mathrm{sec} /$ day $(5-95 \% \mathrm{Cl}=-3.9$ to $3.7 \mathrm{sec} /$ day $)$, latency NTG Trial*ISRIB $2.5 \mathrm{mpk}=0.3$ sec/day $(5-95 \% \mathrm{Cl}=-3.0$ to $3.8 \mathrm{sec} /$ day), latency J20 Trial*ISRIB $0.25 \mathrm{mpk}=-1.4 \mathrm{sec} /$ day $(5-$ $95 \% \mathrm{Cl}=-4.2$ to $6.9 \mathrm{sec} /$ day $)$, latency 220 Trial $*$ ISRIB $2.5 \mathrm{mpk}=4.6 \mathrm{sec} /$ day $(5-95 \% \mathrm{Cl}=-0.8$ to $9.9 \mathrm{sec} / \mathrm{day})$ ). Shaded areas indicate $5-95 \%$ confidence intervals. (E-G) A probe trial was performed 24 hours after completion of the last training trial. (E) NTG mice spent more time in the target quadrant than would be expected by chance, while $J 20$ mice did not (one sample $t$ test against $25 \%$ (dotted line): NTG $1 \%$ DMSO/saline vehicle $p=0.02$, NTG ISRIB $0.25 \mathrm{mpk} p=0.01, \mathrm{~J} 201 \% \mathrm{DMSO} / \mathrm{saline}$ vehicle $p=0.76, \mathrm{~J} 20$ ISRIB $0.25 \mathrm{mpk} p=0.45$, NTG DMSO/PEG vehicle $p=0.0003$, NTG ISRIB $2.5 \mathrm{mpk} p=0.008$, J20 DMSO/PEG vehicle $p=$ 
increase the percentage of time spent in the target quadrant in either group (two-way ANOVA for ISRIB 0.25 mpk: treatment $F_{(1,43)}=0.05, p=0.83$; genotype $F_{(1,43)}=12.88, p=$ 0.0008 ; treatment $x$ genotype $F_{(1,43)}=0.003, p=0.96$. Two-way ANOVA for ISRIB 2.5 mpk: treatment $F_{(1,42)}=1.66, p=0.20$; genotype $F_{(1,42)}=14.68, p=0.0004$; treatment $\times$ genotype $\left.F_{(1,42)}=0.11, p=0.74\right)$. (F) Treatment with ISRIB did not reduce the latency to first cross the target platform location (two-way ANOVA for ISRIB 0.25 mpk: treatment $F_{(1,43)}=0.04, p=$ 0.83; genotype $F_{(1,43)}=7.75, p=0.008$; treatment $x$ genotype $F_{(1,43)}=4.98, p=0.03$. Twoway ANOVA for ISRIB 2.5 mpk: treatment $F_{(1,42)}=1.12, p=0.30$; genotype $F_{(1,42)}=6.62, p=$ 0.01 ; treatment $x$ genotype $F_{(1,42)}=0.26, p=0.61$ ). (G) Treatment with ISRIB did not increase the number of platform crossings (two-way ANOVA for ISRIB $0.25 \mathrm{mpk}$ : treatment $F_{(1,43)}=$ $0.38, p=0.54$; genotype $F_{(1,43)}=8.17, p=0.007$; treatment $\times$ genotype $F_{(1,43)}=0.08, p=$ 0.78. Two-way ANOVA for ISRIB $2.5 \mathrm{mpk}$ : treatment $F_{(1,42)}=0.03, p=0.86$; genotype $F_{(1,42)}=$ 9.97, $p=0.003$; treatment $x$ genotype $\left.F_{(1,42)}=0.77, p=0.39\right)$. $(\mathrm{H}-\mathrm{J})$ A probe trial was performed 72 hours after completion of the last training trial. (H) NTG mice continued to spend more time in the target quadrant compared to chance, except for the group treated with $0.25 \mathrm{mpk}$ ISRIB (one sample $t$ test against 25\% (dotted line): NTG 1\% DMSO/saline vehicle $p=0.02$, NTG ISRIB $0.25 \mathrm{mpk} p=0.23, \mathrm{~J} 201 \% \mathrm{DMSO} / \mathrm{saline}$ vehicle $p=0.48, \mathrm{~J} 20$ ISRIB $0.25 \mathrm{mpk} p=0.73$, NTG DMSO/PEG vehicle $p=0.01$, NTG ISRIB $2.5 \mathrm{mpk} p=0.008, \mathrm{~J} 20$ DMSO/PEG vehicle $p=0.88$, J20 ISRIB $2.5 \mathrm{mpk} p=0.99$ ). No significant treatment effect was observed in either group (two-way ANOVA for ISRIB 0.25 mpk: treatment $F_{(1,43)}=0.23, p=$ 0.63 ; genotype $F_{(1,43)}=4.22, p=0.04$; treatment $x$ genotype $F_{(1,43)}=2.66, p=0.11$. Two-way ANOVA for ISRIB 2.5 mpk: treatment $F_{(1,42)}=0.24, p=0.62$; genotype $F_{(1,42)}=11.85, p=$ 0.001 ; treatment $x$ genotype $F_{(1,42)}=0.13, p=0.72$ ). (I) Treatment with ISRIB did not reduce the latency to first cross the target platform location (two-way ANOVA for ISRIB $0.25 \mathrm{mpk}$ : treatment $F_{(1,43)}=1.44, p=0.24$; genotype $F_{(1,43)}=0.37, p=0.54$; treatment $x$ genotype $F_{(1,43)}$ $=0.40, p=0.53$. Two-way ANOVA for ISRIB $2.5 \mathrm{mpk}$ : treatment $F_{(1,42)}=0.39, p=0.53$; genotype $F_{(1,42)}=19.00, p<0.0001$; treatment $\times$ genotype $\left.F_{(1,42)}=1.28, p=0.26\right)$. (J) 
ISRIB $0.25 \mathrm{mpk}$ : treatment $F_{(1,43)}=4.94, p=0.03$, Sidak's post-hoc comparisons NTG vehicle vs. ISRIB Cl -0.11 to $2.28, \mathrm{~J} 20$ vehicle vs. ISRIB Cl -0.67 to 1.77 ; genotype $F_{(1,43)}=5.31, p=$ 0.03; treatment $x$ genotype $F_{(1,43)}=0.52, p=0.48$. Two-way ANOVA for ISRIB $2.5 \mathrm{mpk}$ : treatment $F_{(1,42)}=0.97, p=0.33$; genotype $F_{(1,42)}=14.02, p=0.0005$; treatment $x$ genotype $\left.F_{(1,42)}=0.0, p>0.99\right) .{ }^{*} p<0.05,{ }^{* *} p<0.01,{ }^{* * *} p<0.001 . \mathrm{Cl}=$ confidence interval, N.S. $=$ not significant, Veh = vehicle, $0.25=$ ISRIB $0.25 \mathrm{mg} / \mathrm{kg}, 2.5=$ ISRIB $2.5 \mathrm{mg} / \mathrm{kg}$, DMSO = dimethyl sulfoxide, PEG = polyethylene glycol. 
APeerJ

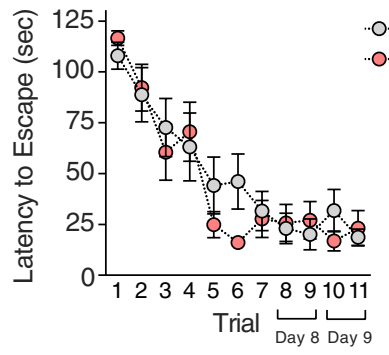

O. NTG 1\%DMSO/saline

O. NTG ISRIB $0.25 \mathrm{mpk}$ 1\%DMSO/saline

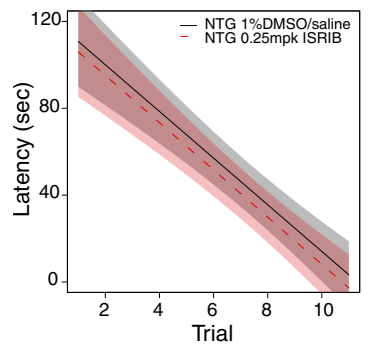

C

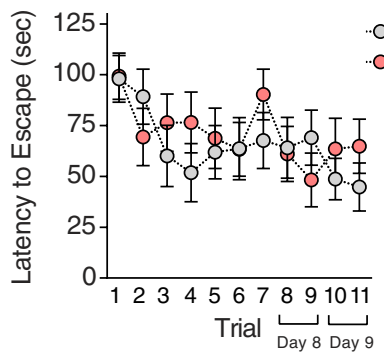

O. J20 1\%DMSO/saline

.. J20 ISRIB $0.25 \mathrm{mpk} 1 \% \mathrm{DMSO} / \mathrm{saline}$

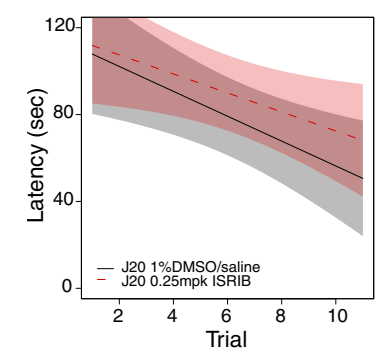

B

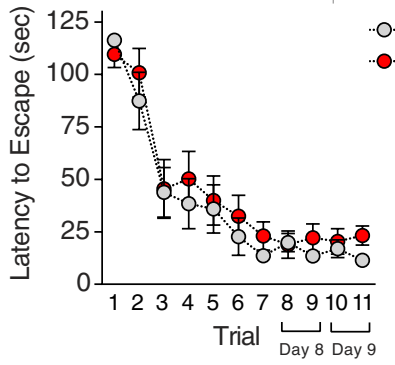

O. NTG DMSO/PEG

. NTG ISRIB 2.5mpk DMSO/PEG

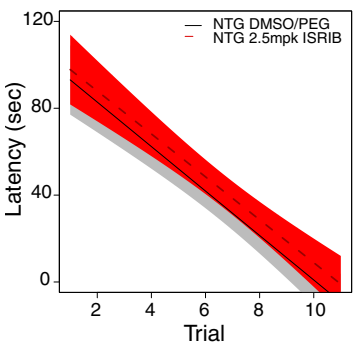

D

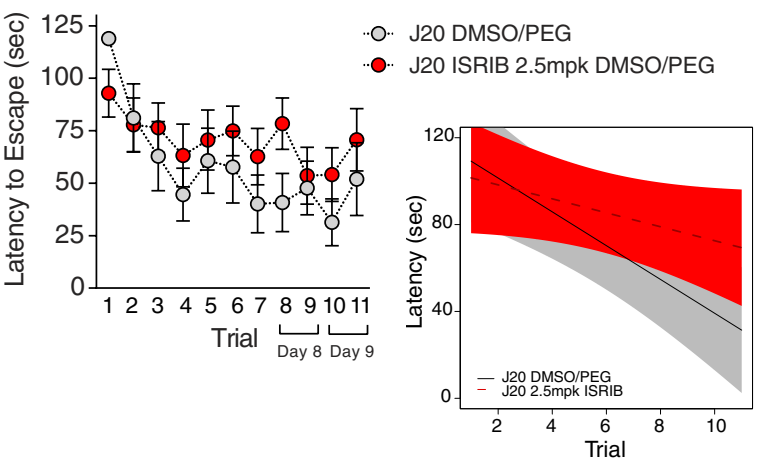

$E$

\% Target Quadrant

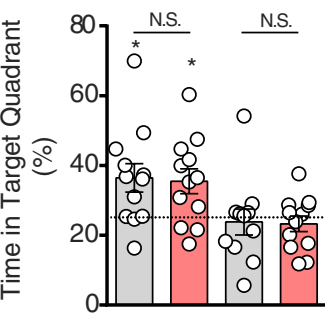

Treatment: Veh 0.25 Veh 0.25

Genotype: NTG

Vehicle: $1 \%$ DMSO/Saline

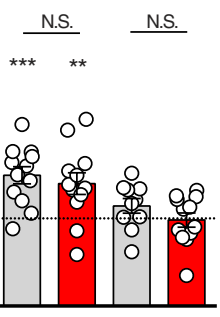

Veh 2.5 Veh 2.5

NTG

F

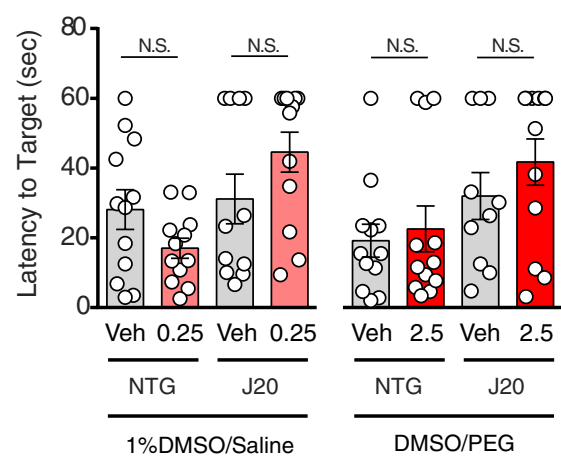

G

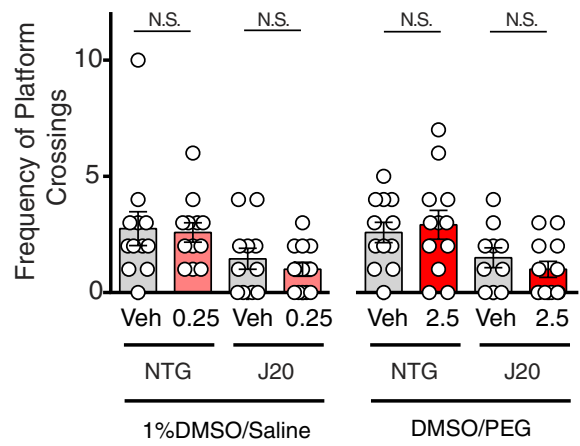

$\mathrm{H}$ 72 hr \% Target Quadrant

$$
\text { 竞 }
$$

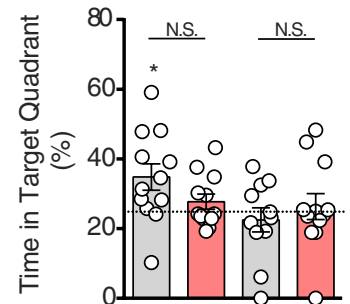

Treatment: Veh 0.25 Veh 0.25

Genotype: NTG J20

Vehicle: $1 \%$ DMSO/Saline

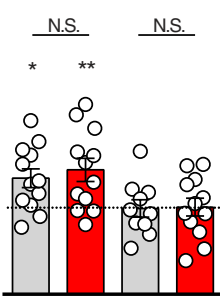

Veh 2.5 Veh 2.5 NTG

DMSO/PEG
$72 \mathrm{hr}$ Latency to Target

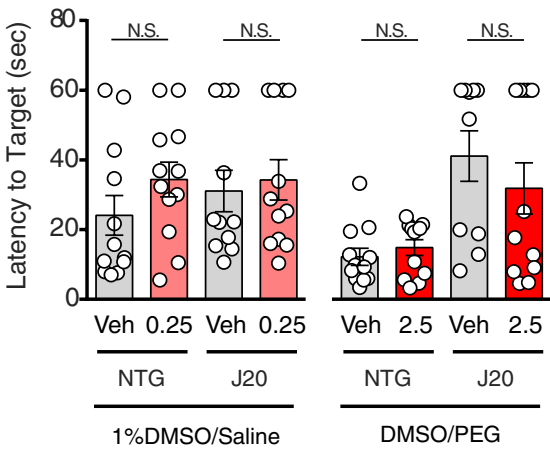

$72 \mathrm{hr}$ Crossing Frequency

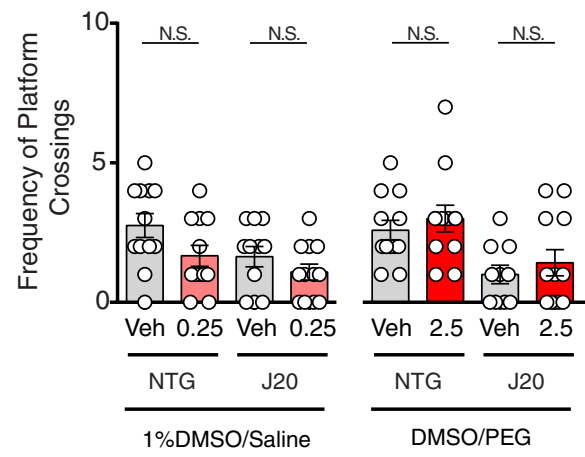

